\title{
A novel alternative splicing isoform of NF2 identified in human Schwann cells
}

\author{
FANG SU, ZHENGGUANG ZHOU, WEN SU, ZISHU WANG and QIONG WU
}

Department of Medical Oncology, The First Affiliated Hospital of Bengbu Medical College, Bengbu, Anhui 233004, P.R. China

Received January 16, 2015; Accepted February 1, 2016

DOI: $10.3892 / 01.2016 .4685$

\begin{abstract}
Vestibular schwannoma (VS) is a benign, slow-growing cranial tumor that originates from the hypertrophy of Schwann cells. The majority of sporadic VS are unilateral, and the mechanisms underlying VS tumorigenesis are not fully understood. The human neurofibromin 2 (NF2) gene encodes the tumor suppressor protein merlin and the NF2 transcript can be alternatively spliced to form numerous isoforms. The present study investigated human Schwann cells (HSCs) at the mRNA and protein level to understand the function of the alternative splicing (AS) isoform of NF2. The total RNA of HSCs was isolated and the full-length coding sequence of NF2 was amplified. The amplified products were excised from agarose gels, purified and sequenced. NF2 at a protein level was assayed by immunoprecipitation and western blot analysis. The full-length and spliced NF2 forms were amplified by polymerase chain reaction (PCR) from the HSC complementary DNA and ligated into eukaryotic expression vector pcDNA3.1(+). The plasmids were transfected into the HSC HEI-193 cell line and cell proliferation assays were performed using Cell Counting Kit-8. PCR analysis using HSC total RNA as a template revealed the presence of a shortened NF2 transcript, which was due to splicing at the 3'-end of the NF2 mRNA. Sequence analysis confirmed that this AS isoform omitted exons 11, 12, 13, 14, 15 and 16. Immunoprecipitation and western blot analysis demonstrated that the AS isoform was highly expressed in the HSCs at $38 \mathrm{kDa}$, while the wild-type (WT) isoform, which was expected at $66 \mathrm{kDa}$, was undetectable. Transfection and cell proliferation assays revealed that the WT isoform exhibited significant growth inhibition, while the AS isoform did not suppress cell growth. In conclusion, the present study detected AS NF2 isoforms in HSC for the first time, and investigated the function of the principle AS isoform. The present study suggests that although
\end{abstract}

Correspondence to: Dr Qiong Wu, Department of Medical Oncology, The First Affiliated Hospital of Bengbu Medical College, 287 Changhuai Road, Bengbu, Anhui 233004, P.R. China

E-mail: qiongwu68@aliyun.com

Key words: vestibular schwannoma, human Schwann cells, NF2, alternative splicing
HSCs have an undetectable level of WT isoform of the NF2 protein merlin, they are not merlin-null, since they express the AS isoform. Although the AS merlin isoform has no suppressive effect on cell growth, certain mechanisms may exist that underlie this phenomenon, and this may be associated with the genesis and development of VS.

\section{Introduction}

Human Schwann cells (HSC) are glial cells located in the peripheral nerves. HSCs undergo proliferation and migration, and assists in neurotrophic support during the development and regeneration of peripheral nerves (1).

Vestibular schwannoma (VS) is a benign, slow-growing cranial tumor. In 1991, the National Institutes of Health Consensus Development Conference reached the conclusion that the term VS should be used rather than acoustic neuroma, since these types of tumor consist of Schwann cells and usually involve the vestibular division of the 8th cranial nerve rather than the acoustic division (2). VS originates from the hypertrophy of Schwann cells, and is divided into two types, namely, unilateral and bilateral tumors (3). The development of bilateral VS is the hallmark of neurofibromatosis type 2 (3), which is an autosomal-dominant tumor predisposition syndrome with an estimated birth incidence of 1 in 33,000 (4). The disease may manifest with deafness, tinnitus and problems with balance. (4). The majority of sporadic VS are unilateral, and this is the most common type of tumor, accounting for $8 \%$ of intracranial tumors (3). Treatment is only by surgical removal. There are numerous clinical symptoms of VS, including sensorineural hearing loss, tinnitus, balance abnormalities, vertigo, facial weakness, blindness, hydrocephalus and even mortality (5).

Although the mechanisms underlying VS tumorigenesis are not fully understood, the human neurofibromin 2 (NF2) gene is known to be associated with VS tumorigenesis. Mutations in NF2 have been identified not only in neurofibromatosis type 2-associated schwannoma, but also in sporadic unilateral VS $(6,7)$. NF2 is located on chromosome $22 q 12$, and although various mutation types, including nonsense, frameshift, missense, insertion, deletion and splice site mutations, have been detected, no mutational hot spots have been previously identified (3).

Biallelic inactivation of NF2 occurs in sporadic and neurofibromatosis type 2 -associated schwannoma, indicating 
that the gene product is a bona fide tumor suppressor (8). NF2 encodes the tumor suppressor protein merlin, and neurofibromatosis type 2-associated schwannoma and sporadic VS are associated with a loss of functional merlin in Schwann cells $(9,10)$.

The NF2 transcript can be alternatively spliced to form numerous isoforms (11) The major isoforms are isoforms I and II, comprising $~ 90 \%$ of mature NF2 transcripts. Other minor alternatively spliced NF2 cDNA variants reported include those missing exons $2,3,8,10$ or 15 , or multiple exons (12). However, only isoform I has been demonstrated to suppress cell growth (13). At present, the role of this post-transcriptional regulation of NF2 mRNA is not well understood.

To understand the function of the NF2 alternative splicing (AS) isoform, the present study investigated HSC at the mRNA and protein level. An AS isoform of NF2 was identified in the HSCs. To identify its function, plasmids were established and the AS isoform was transfected into the VS HEI-193 cell line.

\section{Materials and methods}

Cell culture. This study was approved by the Ethics Committee of The First Affiliated Hospital of Bengbu Medical College (Bengbu, China). HSCs were purchased from ScienCell Research Laboratories (catalog no., 1700; Carlsbad, CA, USA) and maintained in Dulbecco's modified Eagle's medium (DMEM; Invitrogen ${ }^{\circledR}$; Thermo Fisher Scientific, Inc., Waltham, MA, USA), supplemented with $10 \%$ fetal bovine serum (FBS; Invitrogen; Thermo Fisher Scientific, Inc.), $0.1 \%$ penicillin and streptomycin (Invitrogen; Thermo Fisher Scientific, Inc.), and 1\% human Schwann cell supplement (ScienCell Research Laboratories). Human VS HEI-193 cells were obtained from the House Ear Institute (Los Angeles, CA, USA) and were maintained in DMEM supplemented with $10 \%$ FBS and $0.1 \%$ penicillin and streptomycin. All cells were maintained in culture at $37^{\circ} \mathrm{C}$ in an atmosphere of $5 \%$ carbon dioxide. The medium was replaced every 2 days.

RNA extraction and reverse transcription-polymerase reaction (RT-PCR). In total, $10^{6}$ confluent HSCs from 10-cm Petri dishes were washed twice with ice cold phosphate-buffered saline (PBS; $\mathrm{Ca}^{2+} / \mathrm{Mg}^{2+}$-free). Total RNA was isolated using TRIzol ${ }^{\circledR}$ Reagent (Takara Bio, Inc., Otsu, Japan) according to the manufacturer's protocols, and resuspended in diethylpyrocarbonate-treated water. RT-PCR was performed on 500 ng of total RNA using the PrimeScript ${ }^{\circledR}$ RT Reagent kit (Prime Script 1 Step Enzyme Mix; 2x1 Step Buffer; Control F-1 Primer; Control R-1 Primer; Positive Control RNA; RNase Free $\mathrm{dH}_{2} \mathrm{O}$ ) (Takara Bio, Inc.). Synthesis of cDNA was performed at $37^{\circ} \mathrm{C}$ for $15 \mathrm{~min}$ followed by a denaturation step at $85^{\circ} \mathrm{C}$ for $5 \mathrm{sec}$ using the MyCycler PCR Amplifier (Bio-Rad Laboratories, Inc., Hercules, CA, USA).

PCR and analysis of NF2 splicing variants. The full-length coding sequence of NF2 in HSC was amplified using Pfu DNA polymerase (1.25 U/50 $\mu$ l; Promega Corporation, Madison, WI, USA) with the following primers: NF2 forward, 5'-ATAAGA ATGCGGCCGCGCCATGGCCG-3', containing a NotI site (in bold), and reverse, 5'-TGGGCTCTTCACTCAGCTG-3'.
The primers were designed using Primer Premier 5.0 (Premier Biosoft, Palo Alto, CA, USA). The amplification program was as follows: A denaturation step at $95^{\circ} \mathrm{C}$ for $1 \mathrm{~min}$, followed by 30 cycles of amplification $\left(95^{\circ} \mathrm{C}\right.$ for $30 \mathrm{sec}, 62^{\circ} \mathrm{C}$ for $30 \mathrm{sec}$ and $68^{\circ} \mathrm{C}$ for $175 \mathrm{sec}$ ) and a final elongation step at $68^{\circ} \mathrm{C}$ for $8 \mathrm{~min}$. Amplified products were analyzed on $1 \%$ agarose gels (Biowest, Nuaillé, France) using a DNA ladder (DL2000 DNA Marker; Takara Bio, Inc.) and ethidium bromide (BioVision Inc., Milpitas, CA, USA). The expected products were excised from the agarose gels, purified using a Takara Agarose Gel DNA Fragment Recovery kit (Takara Bio, Inc.) and sequenced by Beijing Huada Jierui Biotechnology Co., Ltd. (Beijing, China).

Immunoprecipitation. HSCs $\left(1 \times 10^{6}\right.$ cells $)$ were seeded in $10-\mathrm{cm}$ Petri dishes. Medium was aspirated and the cells were washed twice with cold PBS, and subsequently scraped into lysis buffer [50 mM Tris-buffered saline (TBS; pH 7.5), $150 \mathrm{mM}$ sodium chloride, $1 \mathrm{mM}$ ethylenediaminetetraacetic acid, $0.05 \%$ sodium deoxycholate, $0.1 \%$ sodium dodecyl sulfate (SDS), $60 \mathrm{mM}$ n-octyl- $\beta$-D-glucopyranoside and $1 \%$ Triton X-100] (Takara Bio, Inc.) supplemented with protease and phosphatase inhibitors (Protease Inhibitor Cocktail; Medchem Express LLC, Monmouth, NJ, USA) for $30 \mathrm{~min}$ on ice. The lysate was collected by centrifugation at $13,000 \mathrm{x} g$ for $30 \mathrm{~min}$ at $4^{\circ} \mathrm{C}$, and the supernatant (total cell lysate) was stored at $-80^{\circ} \mathrm{C}$. The protein concentrations were assayed using the Micro BCA Pierce ${ }^{\mathrm{TM}}$ Protein Assay kit (Thermo Fisher Scientific, Inc.). In total, $100 \mathrm{ml}$ total protein lysate was transferred to a $1.5-\mathrm{ml}$ microcentrifuge tube, to which $2 \mu \mathrm{l}$ rabbit polyclonal anti-human NF2 primary antibody (dilution, 1:200; catalog no., sc-331; clone, A-19; Santa Cruz Biotechnology, Inc., Dallas, TX, USA) was added, followed by an incubation time of $1 \mathrm{~h}$ at $4^{\circ} \mathrm{C}$. A $20-\mathrm{ml}$ resuspended volume of Protein A/G PLUS-Agarose (Santa Cruz Biotechnology, Inc.) was then added, and the tubes were capped and incubated at $4^{\circ} \mathrm{C}$ on a rotating device overnight. Immunoprecipitates were collected using centrifugation at 2,500 rpm (1,000 x g) for $5 \mathrm{~min}$ at $4^{\circ} \mathrm{C}$. The supernatant was carefully aspirated and discarded. The pellet was washed 4 times with $1.0 \mathrm{ml}$ lysis buffer, each time repeating the centrifugation step. Following the final wash, the supernatant was aspirated and discarded, and the pellet was resuspended in $40 \mu \mathrm{l} 1 \mathrm{X}$ electrophoresis sample buffer [1 mM Tris- $\mathrm{HCl}(\mathrm{pH} 6.8), 50 \%$ glycerol, $10 \%$ SDS, $0.1 \%$ bromophenol blue and $0.5 \mathrm{ml} \beta$-mercaptoethanol] (Santa Cruz Biotechnology, Inc.). These samples were boiled for $3 \mathrm{~min}$ and $20-\mu \mathrm{l}$ aliquots were subsequently analyzed using SDS-polyacrylamide gel electrophoresis (SDS-PAGE) and autoradiography. Unused samples were stored at $-20^{\circ} \mathrm{C}$.

Western blot analysis. The protein lysate was resolved using 8-12\% SDS-PAGE and transferred to immobilon polyvinylidene difluoride membranes (Bio-Rad Laboratories, Inc.). The membranes were blocked with blocking buffer (5\% skimmed dried milk and $0.1 \%$ Tween 20 in TBS) for $1 \mathrm{~h}$ at room temperature, and subsequently incubated with primary polyclonal rabbit anti-mouse $\beta$-actin (catalog no., sc-130656; dilution, 1:200; Santa Cruz Biotechnology, Inc.) and NF2 antibodies (catalog no., sc-331; dilution, 1:200; Santa Cruz Biotechnology, Inc.) overnight at $4^{\circ} \mathrm{C}$. The membranes were 
A

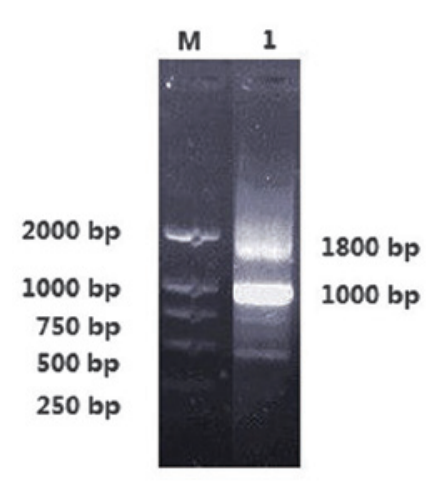

B

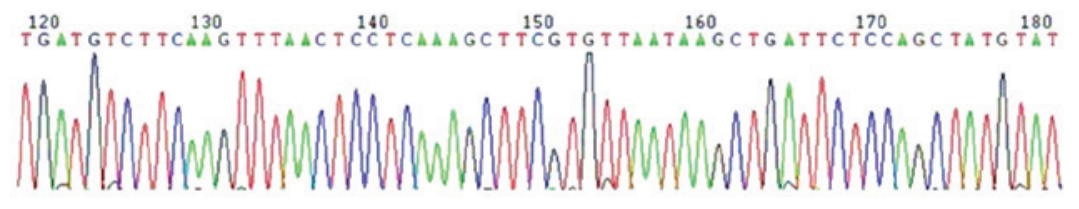

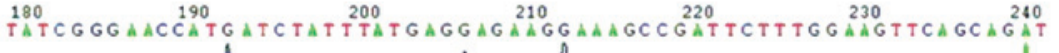

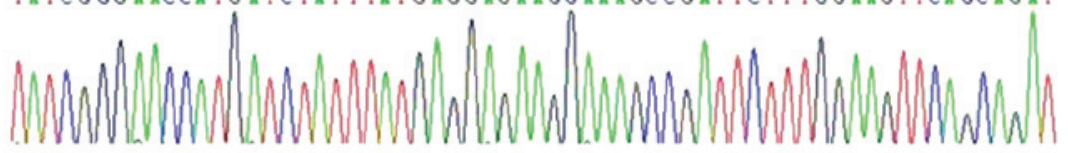

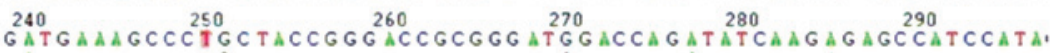

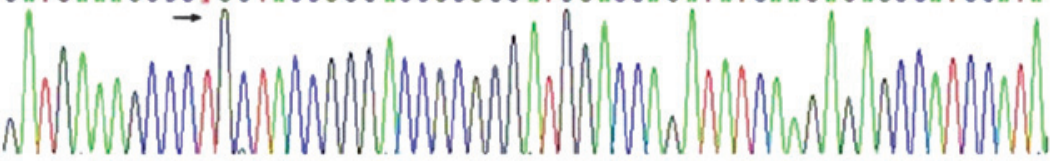

C

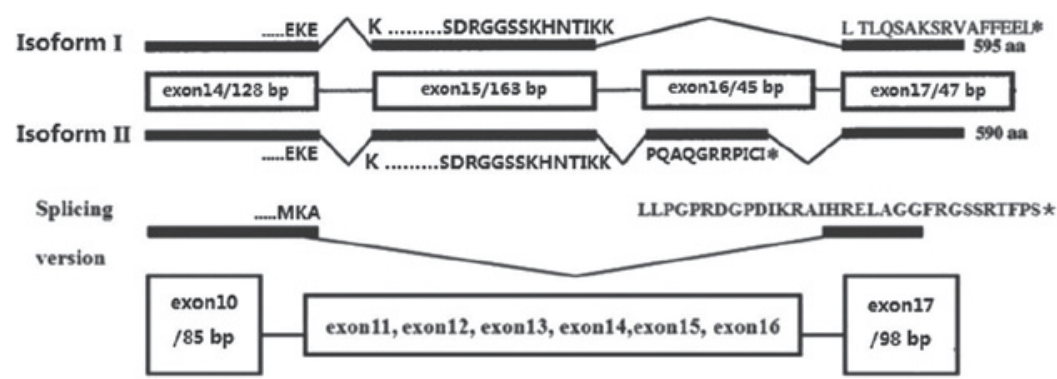

Figure 1. (A) Lane 1, polymerase chain reaction product of NF2 in human Schwann cells. (B) Partial sequence of AS NF2. The arrow indicates the splicing site. (C) The translational consequence of NF2 alternative splicing transcripts. Previously identified COOH-terminal isoforms I and II resulting from alternative splicing of exons 15, 16 and 17. The novel AS isoform contains the first 10 exons and connects to exon 17. The COOH-terminus of the novel AS isoform differs from isoform I and II. The number of residues contained in each polypeptide is indicated. * indicates the Merlin COOH-terminus. M, DNA marker DL2000; AS, alternative splicing.

then washed 3 times with washing buffer [1X TBS $(0.1 \%)$ and Tween 20] for 10 min and subsequently incubated with mouse anti-rabbit immunoglobulin $\mathrm{G}$ horseradish peroxidase-conjugated secondary antibody (catalog no., sc-2357; dilution, 1:5,000; Santa Cruz Biotechnology, Inc.) for $2 \mathrm{~h}$ at room temperature. Following 3 additional washes, the antibody was detected using a Clarity ${ }^{\mathrm{TM}}$ Enhanced Chemiluminescence Western Blot Substrate kit (Bio-Rad Laboratories, Inc.) and autoradiography using X-ray film. $\beta$-actin was included as a loading control.

Plasmid construction and DNA transfection. The full-length and spliced NF2 cDNA from normal HSCs were amplified using the aforementioned PCR process. Detection primers were as follows: WT isoform forward, 5'-GCCCATGAA CCCAATTCCAGCAC-3' and reverse, 5'-TGAGCTGCTCCT GCAGATGCTTG-3'; and AS isoform forward, 5'ATAGCT CAGGACCTGGAGATG-3' and reverse, 5'-TGGGCTCTT CACTCAGCTG-3'. The primers were designed using Primer Premier 5.0. The eukaryotic expression vector pcDNA3.1(+) (Invitrogen; Thermo Fisher Scientific, Inc.) and wild-type (WT) NF2 and AS NF2 purified products were digested using NotI and XbaI. The resulting products were ligated with T4 DNA Ligase (New England BioLabs, Inc., Ipswich, MA, USA), and transformed into DH5 $\alpha$ strains of Escherichia coli (Tiangen Biotech Co., Ltd., Beijing, China). Extracted recombinant plasmids were identified and analyzed using a restrictive endonuclease digestion map and sequence analysis software
(Primer Premier 5.0; Premier Biosoft International, Palo Alto, CA, USA). The novel recombinant plasmids were termed pcDNA3.1-(WT)-NF2 and pcDNA3.1-(AS)-NF2. These plasmids were transiently transfected into HEI-193 cells using Lipofectamine ${ }^{\circledR} 2000$ (Invitrogen; Thermo Fisher Scientific, Inc.) in 6 -cm plates and 96 -well plates. The cells were harvested at $72 \mathrm{~h}$ post-transfection. Total DNA was purified from the cells using DNAiso Reagent (Takara Bio, Inc.).

Cell proliferation assay. Cell proliferation assays were performed using Cell Counting Kit-8 (CCK-8; Dojindo Molecular Technologies, Inc., Kumamoto, Japan). HEI-193 cells were plated $24 \mathrm{~h}$ prior to the assay in 96-well plates at a density of $1 \times 10^{4}$ cells/well and cultured in the growth medium. The recombinant plasmids, pcDNA3.1-WT-NF2 and pcDNA3.1-AS-NF2, were applied to the cells at the indicated concentrations; $0.2 \mu \mathrm{g}$ of each plasmid was added per well in the 96-well plates, and $4 \mu \mathrm{g}$ of each plasmid was addded to the $6-\mathrm{cm}$ plates. Subsequent to transfection for 24 , 48 and 72 h, WST-8 was added to the cells and cell numbers in triplicate wells were measured at an absorbance of $450 \mathrm{~nm}$.

Statistical analysis. Statistical analysis was performed using SPSS version 11.0 (SPSS, Inc., Chicago, IL, USA) and one-way single factor analysis of variance. Data are from three experimental repeats in all groups. Fisher's exact test was used to analyze categorical data. Student-Newman-Keuls 
A

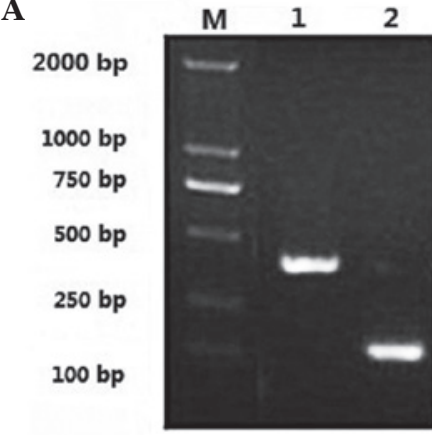

B

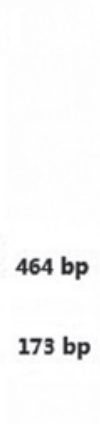

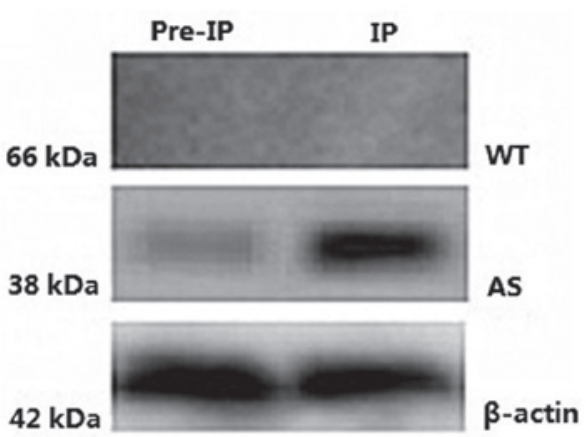

C

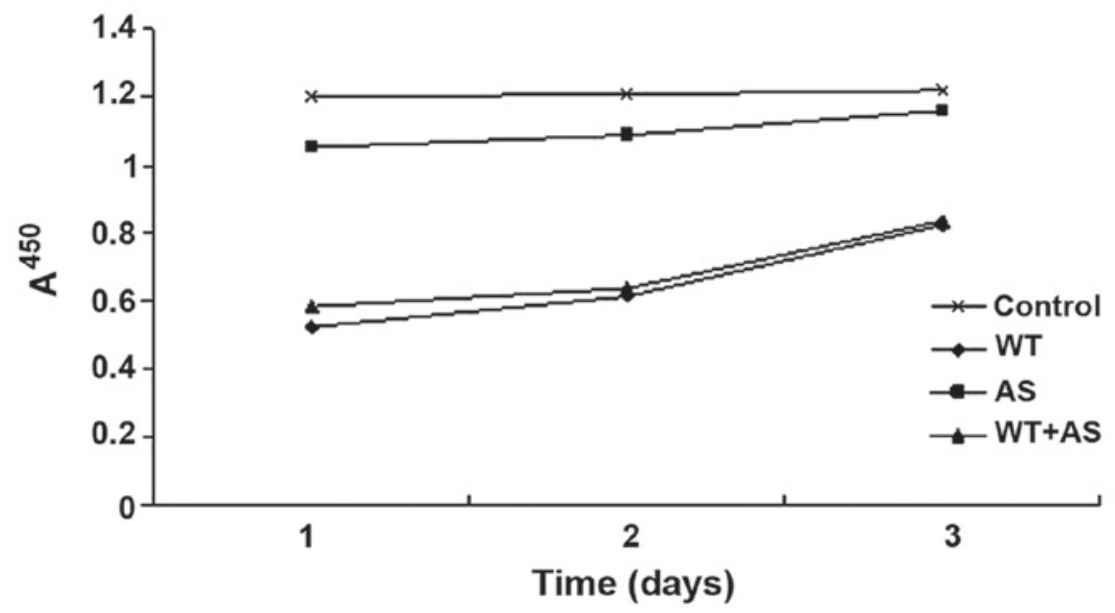

Figure 2. (A) Effect of transfection of human vestibular schwannoma HEI-193 cells. Lane 1, transfected HEI-193 cells with plasmid encoding AS NF2. Lane 2, transfected HEI-193 cells with plasmid encoding WT NF2. (B) Levels of NF2 protein in HSCs: The AS isoform was highly expressed in HSCs at 38 kDa, while the WT isoform, which was expected at $66 \mathrm{kDa}$, was undetectable. (C) The novel AS NF2 isoform had no suppressive effect on cell proliferation. HEI-193 cells were transfected with recombinant plasmids expressing WT, AS NF2 or the two isoforms. The effect of the expression of these isoforms on cell proliferation was measured using a cell counting kit-8 assay for 3 days. M, DNA marker DL2000; AS, alternative splicing; WT, wild-type; HSC, human Schwannoma cells; Ctrl, control; $\mathrm{A}^{450}$, absorbance at $450 \mathrm{~nm}$; IP, immunoprecipitation.

test was used to conduct pairwise comparisons among the three groups. $\mathrm{P}<0.05$ was used to indicate that total comparison differences were statistically significant, and $\mathrm{P}<0.01$ was used to indicate that pair-wise comparisons among groups were statistically significant.

\section{Results}

Characterization of NF2 transcripts present in HSCs. In order to confirm the presence of NF2 transcript alterations in HSCs, the present study examined NF2 gene expression in HSCs by PCR analysis using HSC total RNA as a template. As shown in Fig. 1A, the upper NF2 transcript from the HSCs was $1.8 \mathrm{~kb}$, as predicted, encompassing a full-length open reading frame, whereas the lower transcript was shorter at $1.0 \mathrm{~kb}$. The smaller bands reflect other AS transcripts, which are commonly observed in numerous cell types (14). The two principle fragments underwent sequence analysis and a region of the AS isoform sequence is shown in Fig. 1B. Sequence analysis revealed that the AS isoform in the HSCs omitted exons 11, 12, 13, 14, 15 and 16 during processing of the NF2 pre-mRNA, resulting in a shortened NF2 transcript. A sketch map is shown in Fig. 1C.

Taken together, these data suggested that the NF2 AS isoform in HSCs results in a shortened NF2 transcript due to splicing at the 3 '-end of the NF2 mRNA.
Effect of transfection of HEI-193 cells with constructed plasmids. To investigate the function of the constructed plasmids, the present study transfected HEI-193 cells with plasmids encoding WT or AP NF2, or the two in combination. Fig. 2A shows that when ectopically expressed in the HEI-193 cell line, the AS isoform is as efficiently expressed as the WT isoform. The results indicated that the HEI-193 cells are readily amenable to transfection.

Characterization of the NF2 protein merlin in HSCs. Nonsense mutations and small base pair insertions/deletions may result in truncated proteins (15). To investigate whether a truncated merlin protein was present in the HSCs, the present study extracted proteins from the HSCs, and the lysates were subjected to immunoprecipitation using the polyclonal anti-NF2 antibody. Subsequent immunoblot analysis using the same antibody revealed that the AS isoform was highly expressed in HSCs at $38 \mathrm{kDa}$, consistent with the molecular weight calculated from the amino acid sequence (Fig. 2B). However, the WT isoform, which was expected at $66 \mathrm{kDa}$, was undetectable. This indicates that although HSCs have an undetectable level of WT merlin isoform, they are, in fact, not merlin-null, since they express the AS isoform.

Growth inhibition of HEI-193 cells by recombinant plasmids. The AS at the 3'-end of WT NF2 leads to the replacement of 
the 16 amino acid $\mathrm{C}$-terminus of isoform $\mathrm{I}$ with the 11 amino acid C-terminus of isoform II (Fig. 1C). This difference is apparently sufficient to prevent isoform II from being growth suppressive (16). To determine whether the AS isoform possesses growth suppressing activity, the HEI-193 cells were transfected with recombinant plasmids expressing the WT or $\mathrm{AS}$ isoforms, or the two isoforms in combination, and their effect on cell proliferation was measured using a CCK-8 assay. As shown in Fig. 2C, the cells transfected with the WT isoform demonstrated a significant cell growth inhibitory effect. By contrast, the cells transfected with the AS isoform did not affect cell growth inhibition $(\mathrm{P}>0.05)$. Therefore, the present study concluded that unlike the WT isoform, the AS isoform does not suppress cell growth.

\section{Discussion}

The human VS HEI-193 cell line was derived from a patient with neurofibromatosis type 2 presenting with spontaneous bilateral VS and a history of meningioma (17). The schwannomas of the patient were resected and the tumor cells were immortalized using the human papilloma virus E6-E7 genes (18). NF2-deficient cells and animal models have contributed significantly to the understanding of the function of the merlin protein. The significance of merlin in cell growth regulation and development has been previously demonstrated by targeted knockdown of NF2, which resulted in the mortality of embryos at day 6.5 in pregnant mice (19), whereas in NF2 heterozygous mice the development of a variety of highly metastatic types of tumor was observed (20). The first studies that used primary cultured NF2-deficient schwannoma cells isolated from human tumors found unique mutant phenotypic traits at the cellular level, such as cytoskeletal abnormalities (21) and growth characteristics (22). However, these cells grow extremely slowly and it is challenging to transfect them with DNA, therefore rendering biochemical and mechanistic studies difficult (23). By contrast, the HEI-193 cell line does not have these disadvantages.

By sequencing the AS isoform of the NF2 locus from HSCs, the present study confirmed the existence of a novel isoform of the NF2 gene sequence. This splicing mutation omitted exons $11,12,13,14,15,16$ and a region of 17 . Due to novel splicing, the amino acid sequence of this splice variant is significantly shorter and differs completely from isoforms I or II, which are located near the C-terminus. Unexpectedly, the amino acid sequence is distinct from any merlin isoforms described previously (24).

HSCs are normal cells located in the peripheral nerves. Using PCR, the present study detected the existence of an AS isoform of NF2 in HSCs. Western blot analysis revealed that the merlin protein expressed by the HSCs was $38 \mathrm{kDa}$ in size, which was consistent with the PCR results. Therefore, the a plasmid was constructed with the principle NF2 AS isoform and it was transfected into the HEI-193 cells.

HSCs, which exclusively express the novel truncated protein, may provide the opportunity to determine the suppressive effect on cell growth of the novel AS isoform. The present cell proliferation studies using HEI-193 cells transfected with plasmids encoding the WT isoform, AS isoform or the two isoforms in combination, provided a direct comparison between the growth-suppressive activity of the isoforms, and revealed that unlike the WT isoform, the novel AS isoform does not suppress cell growth. These results provide experimental validation of the hypothesis that the AS isoform does not have a suppressive effect on cell growth. The results also suggest that although HSCs lack the WT isoform of merlin, they are not merlin-null, since they express the AS isoforms of merlin.

In conclusion, to the best of our knowledge, the present study identified the novel AS NF2 isoforms in HSC for the first time, and investigated the function of the principle AS isoform identified. Although it has no effect on cell growth suppression, it is notable that such a normal cell line has significant AS isoforms. A mechanism may exist that underlies this phenomenon, and this may be associated with the genesis and development of VS. Further research is required for elucidation.

\section{Acknowledgements}

The present study was supported by the Natural Science Research Program funded by the Education Department of Anhui Province (grant no. KJ2013B145).

\section{References}

1. Mahanthappa NK, Anton ES and Matthew WD: Glial growth factor 2, a soluble neuregulin, directly increases Schwann cell motility and indirectly promotes neurite outgrowth. J Neurosci 16: 4673-4683, 1996.

2. Shepard TH, Tucci DL, Grant GA and Kaylie DM: Management of hearing in pediatric NF2. Otol Neurotol 33: 1066-1070, 2012.

3. Diensthuber M, Lenarz T and Stöver T: Neurotrophic factor expression in vestibular schwannoma. An overview. Laryngorhinootologie 85:731-737, 2006 (In German).

4. Hexter A, Jones A, Joe H, Heap L, Smith MJ, Wallace AJ, Halliday D, Parry A, Taylor A, Raymond L, et al: Clinical and molecular predictors of mortality in neurofibromatosis 2 : A UK national analysis of 1192 patients. J Med Genet 52: 699-705, 2015.

5. Drouet A, Le Moigne F, Salamé D, Quesnel L, Motolese C, des Portes V, Guilloton L and Pinson S: Type 2 neurofibromatosis: Intergenerational differences in genetic and clinical expression. Arch Pediatr 21: 1233-1240, 2014 (In French).

6. Chen H, Zhang X, Zhang Z, Yang T, Wang Z and Wu H: The role of NF2 gene mutations and pathogenesis-related proteins in sporadic vestibular schwannomas in young individuals. Mol Cell Biochem 392: 145-152, 2014.

7. Kuga Y, Ohnishi H, Kodama Y, Takakura S, Hayashi M, Yagi R, Fukutome K, Matsushima K, Okamoto K, Taomoto K and Takahashi H: Cerebral and spinal cord tanycytic ependymomas in a young adult with a mutation in the NF2 gene. Neuropathology 34: 406-413, 2014.

8. Harvey KF, Zhang X and Thomas DM: The Hippo pathway and human cancer. Nat Rev Cancer 13: 246-257, 2013.

9. Petrilli A, Copik A, Posadas M, Chang LS, Welling DB Giovannini $\mathrm{M}$ and Fernández-Valle C: LIM domain kinases as potential therapeutic targets for neurofibromatosis type 2 . Oncogene 33: 3571-3582, 2014

10. Li W, Cooper J, Karajannis MA and Giancotti FG: Merlin: A tumour suppressor with functions at the cell cortex and in the nucleus. EMBO Rep 13: 204-215, 2012.

11. Schmucker B, Tang Y and Kressel M: Novel alternatively spliced isoforms of the neurofibromatosis type 2 tumor suppressor are targeted to the nucleus and cytoplasmic granules. Hum Mol Genet 8: 1561-1570, 1999

12. Rustgi AK, Xu L, Pinney D, Sterner C, Beauchamp R, Schmidt S, Gusella JF and Ramesh V: Neurofibromatosis 2 gene in human colorectal cancer. Cancer Genet Cytogenet 84: 24-26, 1995.

13. Lepont P, Stickney JT, Foster LA, Meng JJ, Hennigan RF and Ip W: Point mutation in the NF2 gene of HEI-193 human schwannoma cells results in the expression of a merlin isoform with attenuated growth suppressive activity. Mutat Res 637: 142-1451, 2008. 
14. Chang LS, Akhmametyeva EM, Wu Y, Zhu L and Welling DB: Multiple transcription initiation sites, alternative splicing and differential polyadenylation contribute to the complexity of human neurofibromatosis 2 transcripts. Genomics 79: 63-76, 2002.

15. Barker CL, Baillie BK, Hammond-Kosack KE, Jones JD and Jones DA: Dominant-negative interference with defence signalling by truncation mutations of the tomato Cf- 9 disease resistance gene. Plant J 46: 385-399, 2006.

16. Doherty JK, Ongkeko W, Crawley B, Andalibi A and Ryan AF: ErbB and Nrg: Potential molecular targets for vestibular schwannoma pharmacotherapy. Otol Neurotol 29: 50-57, 2008.

17. Angelo LS, Maxwell DS, Wu JY, Sun D, Hawke DH, McCutcheon IE, Slopis JM, Peng Z, Bornmann WG and Kurzrock R: Binding partners for curcumin in human schwannoma cells: Biologic implications. Bioorg Med Chem 21: 932-939, 2013

18. Prabhakar S, Taherian M, Gianni D, Conlon TJ, Fulci G, Brockmann J, Stemmer-Rachamimov A, Sena-Esteves M, Breakefield XO and Brenner GJ: Regression of schwannomas induced by adeno-associated virus-mediated delivery of caspase- 1 . Hum Gene Ther 24: 152-162, 2013.
19. Cockburn K, Biechele S, Garner J and Rossant J: The Hippo pathway member Nf2 is required for inner cell mass specification. Curr Biol 23: 1195-1201, 2013.

20. Cačev T, Aralica G, Lončar B and Kapitanović S: Loss of NF2/Merlin expression in advanced sporadic colorectal cancer. Cell Oncol (Dordr) 37: 69-77, 2014.

21. Thurneysen C, Opitz I, Kurtz S, Weder W, Stahel RA and Felley-Bosco E: Functional inactivation of NF2/merlin in human mesothelioma. Lung Cancer 64: 140-147, 2009.

22. Kros J, de Greve K, van Tilborg A, Hop W, Pieterman H, Avezaat C, Lekanne Dit Deprez R and Zwarthoff E: NF2 status of meningiomas is associated with tumour localization and histology. J Pathol 194: 367-372, 2001.

23. Dilwali S, Patel PB, Roberts DS, Basinsky GM, Harris GJ, Emerick KS and Stankovic KM: Primary culture of human Schwann and schwannoma cells: Improved and simplified protocol. Hear Res 315: 25-33, 2014.

24. Sainio M, Jääskeläinen J, Pihlaja $\mathrm{H}$ and Carpén O: Mild familial neurofibromatosis 2 associates with expression of merlin with altered COOH-terminus. Neurology 54: 1132-1138, 2000. 